\title{
Terminology and acronyms
}

\begin{tabular}{|c|c|}
\hline ADR & $\begin{array}{l}\text { American depository receipt (vehicle for trading } \\
\text { non-American stocks on American stock exchanges) }\end{array}$ \\
\hline AGM & annual general meeting (gathering of all shareholders) \\
\hline APG & associated petroleum gas (gas found along with oil) \\
\hline $\mathrm{ARCO}$ & Atlantic Richfield Company \\
\hline BCG & Boston Consulting Group \\
\hline billion & a thousand million \\
\hline $\mathrm{BP}$ & (formerly) British Petroleum \\
\hline CEO & chief executive officer \\
\hline CIS & Commonwealth of Independent States \\
\hline $\mathrm{CNPC}$ & China National Petroleum Corporation \\
\hline CSR & corporate social responsibility \\
\hline $\mathrm{E} \& \mathrm{P}$ & exploration and production \\
\hline EITI & Extractive Industries Transparency Initiative \\
\hline EOL & Essar Oil Limited \\
\hline ESPO & Eastern Siberia-Pacific Ocean pipeline \\
\hline EU & European Union \\
\hline FDI & foreign direct investment \\
\hline GAAP & generally accepted accounting principles \\
\hline GDP & gross domestic product \\
\hline GHG & greenhouse gas \\
\hline GRI & Global Reporting Initiative \\
\hline HSE & health, safety and environment \\
\hline IAS & international accounting standards \\
\hline IEA & International Energy Agency \\
\hline IFRS & international financial reporting standards \\
\hline IOC & international oil company \\
\hline
\end{tabular}




$\begin{array}{ll}\text { IPO } & \text { initial public offering } \\ \text { IRENA } & \text { International Renewable Energy Agency } \\ \text { IT } & \text { information technology } \\ \text { JSC } & \text { joint stock company } \\ \text { KNOC } & \text { Korean National Oil Corporation } \\ \text { LNG } & \text { liquefied natural gas } \\ \text { LSE } & \text { London Stock Exchange } \\ \text { M\&A } & \text { mergers and acquisitions } \\ \text { MD\&A } & \text { management discussion and analysis } \\ \text { MoU } & \text { memorandum of understanding } \\ \text { MRH } & \text { Mineralol Rohstoff Handel } \\ \text { NFK } & \text { Neftyanaya Finansovaya Kompaniya } \\ \text { NIOC } & \text { National Iranian Oil Company } \\ \text { NIS } & \text { Naftna Industrija Srbije } \\ \text { NOC } & \text { national oil company } \\ \text { NYSE } & \text { New York Stock Exchange } \\ \text { OECD } & \text { Organisation for Economic Co-operation and } \\ \text { OJSC } & \text { Development } \\ \text { ONACO } & \text { open joint stock company } \\ \text { ONGC } & \text { Orenburgskaya Neftyanaya Kompaniya (Orenburg Oil } \\ \text { OPEC } & \text { Company) } \\ \text { PDVSA } & \text { Oil and Natural Gas Corporation (India) } \\ \text { PSA } & \text { Organization of Petroleum Exporting Countries } \\ \text { R\&D } & \text { Petróleos de Venezuela } \\ \text { Rosnedra } & \text { production-sharing agreement } \\ \text { R/P } & \text { research and development } \\ \text { RTS } & \text { Federalnoe agenstvo po nedropolzovaniyu (Federal } \\ \text { RUB } & \text { Agency for Subsoil Use) } \\ \text { S\&P } & \text { reserves to production ratio } \\ \text { SEC } & \text { Russian Trading System } \\ & \text { Russian roubles } \\ \text { Standard and Poor's Financial Services } \\ \text { Securities and Exchange Commission } \\ \end{array}$


SIDANCO Sibirskaya-dalnevostochnaya Neftyanaya Kompaniya (Siberian-Far Eastern Oil Company)

TNK Tyumenskaya Neftyanaya Kompaniya (Tyumen Oil Company)

TRN

Total Refinery Netherlands

TSR

total shareholder return

UN

United Nations

UNESCO United Nations Educational, Scientific and Cultural Organization

USSR Union of Soviet Socialist Republics

WWF World Wildlife Fund

YUNKO Yuzhnaya Neftyanaya Kompaniya (Southern Oil Company) 